NASA/TM-2008-214829

\title{
SmaggIce 2.0: Additional Capabilities for Interactive Grid Generation of Iced Airfoils
}

Richard E. Kreeger, Marivell Baez, Donald C. Braun, Herbert W. Schilling, and Mary B. Vickerman

Glenn Research Center, Cleveland, Ohio 


\section{NASA STI Program . . . in Profile}

Since its founding, NASA has been dedicated to the advancement of aeronautics and space science. The NASA Scientific and Technical Information (STI) program plays a key part in helping NASA maintain this important role.

The NASA STI Program operates under the auspices of the Agency Chief Information Officer. It collects, organizes, provides for archiving, and disseminates NASA's STI. The NASA STI program provides access to the NASA Aeronautics and Space Database and its public interface, the NASA Technical Reports Server, thus providing one of the largest collections of aeronautical and space science STI in the world. Results are published in both non-NASA channels and by NASA in the NASA STI Report Series, which includes the following report types:

- TECHNICAL PUBLICATION. Reports of completed research or a major significant phase of research that present the results of NASA programs and include extensive data or theoretical analysis. Includes compilations of significant scientific and technical data and information deemed to be of continuing reference value. NASA counterpart of peer-reviewed formal professional papers but has less stringent limitations on manuscript length and extent of graphic presentations.

- TECHNICAL MEMORANDUM. Scientific and technical findings that are preliminary or of specialized interest, e.g., quick release reports, working papers, and bibliographies that contain minimal annotation. Does not contain extensive analysis.

- CONTRACTOR REPORT. Scientific and technical findings by NASA-sponsored contractors and grantees.
- CONFERENCE PUBLICATION. Collected papers from scientific and technical conferences, symposia, seminars, or other meetings sponsored or cosponsored by NASA.

- SPECIAL PUBLICATION. Scientific, technical, or historical information from NASA programs, projects, and missions, often concerned with subjects having substantial public interest.

- TECHNICAL TRANSLATION. Englishlanguage translations of foreign scientific and technical material pertinent to NASA's mission.

Specialized services also include creating custom thesauri, building customized databases, organizing and publishing research results.

For more information about the NASA STI program, see the following:

- Access the NASA STI program home page at http://www.sti.nasa.gov

- E-mail your question via the Internet to help@sti.nasa.gov

- Fax your question to the NASA STI Help Desk at 301-621-0134

- Telephone the NASA STI Help Desk at 301-621-0390

- Write to: NASA Center for AeroSpace Information (CASI) 7115 Standard Drive Hanover, MD 21076-1320 
NASA/TM-2008-214829

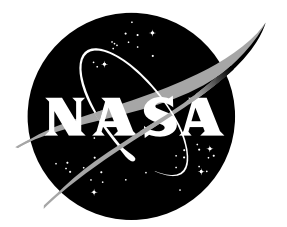

\section{SmaggIce 2.0: Additional Capabilities for Interactive Grid Generation of Iced Airfoils}

Richard E. Kreeger, Marivell Baez, Donald C. Braun, Herbert W. Schilling, and Mary B. Vickerman

Glenn Research Center, Cleveland, Ohio

Prepared for the

45th Aerospace Sciences Meeting and Exhibit

sponsored by the American Institute of Aeronautics and Astronautics

Reno, Nevada, January 8-11, 2007

National Aeronautics and

Space Administration

Glenn Research Center

Cleveland, Ohio 44135 
Level of Review: This material has been technically reviewed by technical management.

Available from

NASA Center for Aerospace Information

7115 Standard Drive

Hanover, MD 21076-1320
National Technical Information Service 5285 Port Royal Road Springfield, VA 22161

Available electronically at http://gltrs.grc.nasa.gov 


\title{
SmaggIce 2.0: Additional Capabilities for Interactive Grid Generation of Iced Airfoils
}

\author{
Richard E. Kreeger, Marivell Baez, Donald C. Braun, Herbert W. Schilling, and Mary B. Vickerman \\ National Aeronautics and Space Administration \\ Glenn Research Center \\ Cleveland, Ohio 44135
}

\begin{abstract}
The Surface Modeling and Grid Generation for Iced Airfoils (SmaggIce) software toolkit has been extended to allow interactive grid generation for multi-element iced airfoils. The essential phases of an icing effects study include geometry preparation, block creation and grid generation. SmaggIce Version 2.0 now includes these main capabilities for both single and multi-element airfoils, plus an improved flow solver interface and a variety of additional tools to enhance the efficiency and accuracy of icing effects studies. An overview of these features is given, especially the new multi-element blocking strategy using the multiple wakes method. Examples are given which illustrate the capabilities of SmaggIce for conducting an icing effects study for both single and multi-element airfoils.
\end{abstract}

$\begin{array}{ll}2 D & \text { two-dimensional } \\ 3 D & \text { three-dimensional } \\ C & \text { chord length } \\ C F D & \text { computational fluid dynamics } \\ \text { CGNS } & \text { CFD General Notation System } \\ \text { LWC } & \text { liquid water content, } \mathrm{g} / \mathrm{m}^{3} \\ \text { MVD } & \text { median volume diameter, } \mu \mathrm{m} \\ \text { SmaggIce } & \text { Surface Modeling and Grid Generation for Iced Airfoils } \\ X & \text { x-coordinate } \\ \text { Xle } & \text { x-coordinate of leading edge }\end{array}$

\section{Introduction}

$\mathrm{T}$ wo types of problems are usually considered in icing: ice accretion and icing effects. Ice accretion geometries change over time in a quasi-steady manner, which requires frequent grid modifications, and is more suited to automated procedures. Icing effects, on the other hand, seek to determine the flow field and performance degradation given an ice shape, and so are more suited to interactive tools. Any computational icing effects study must address the issues of ice shape characterization, grid generation, and aerodynamic flow solution.

The Surface Modeling and Grid Generation for Iced Airfoils (SmaggIce) toolkit is a suite of interactive tools developed at the NASA Glenn Research Center ${ }^{1,2}$ which can simplify and improve an icing effects study. This suite of tools is used to prepare two-dimensional cross sections of iced airfoils for computational fluid dynamics (CFD) analysis. SmaggIce is designed to help researchers and engineers study the effects of ice accretion on airfoil performance, which is difficult to do with other software packages because of the complexity of ice shapes ${ }^{3}$. CFD tools are used primarily for certification studies and analysis, to evaluate safety of flight with unprotected surfaces and to determine the need for ice protection. They are also used for the design and analysis of ice protection systems. Using SmaggIce to simulate flow over an iced airfoil can help to reduce the cost of performing flight and 
wind-tunnel tests for certifying aircraft in natural and simulated icing conditions. The phases of a computational icing effects study are shown in Figure 1.

Evaluation of the ice geometry includes identification of potential geometric problems. Ice shapes pose difficulty in generating the good-quality grids that are essential for predicting ice-induced complex flow. Ice geometries can come from experimental or predicted sources. Ice shape characterization gives a quantitative assessment and comparisons of ice shapes. SmaggIce provides tools to rapidly quantify ice-shape characteristics (such as horn height, angle and location) and calculate integrated ice area, allowing researchers to examine their effect on aerodynamic performance. It includes tools that examine input data for possible errors (such as tangling introduced during data acquisition) and allows the user to smooth ice shapes to desired levels for computational fluid dynamics analysis. There is a third category of use to the engineer: artificial ice. SmaggIce allows users to specify the size and location of simple, primitive ice shapes for parametric study.

The SmaggIce tools are uniquely tailored for icing effects studies, including dividing the flow domain into blocks to set up the grid structure prior to grid generation, making changes to the grid-density distribution, and merging and smoothing multi-block grids. The use of a thin, tightly controlled block that wraps around the iced airfoil is an example of one of many unique features available for handling difficult ice geometries. The shapes of block edges can be changed via control points. The density and distribution of points over the iced airfoil can be

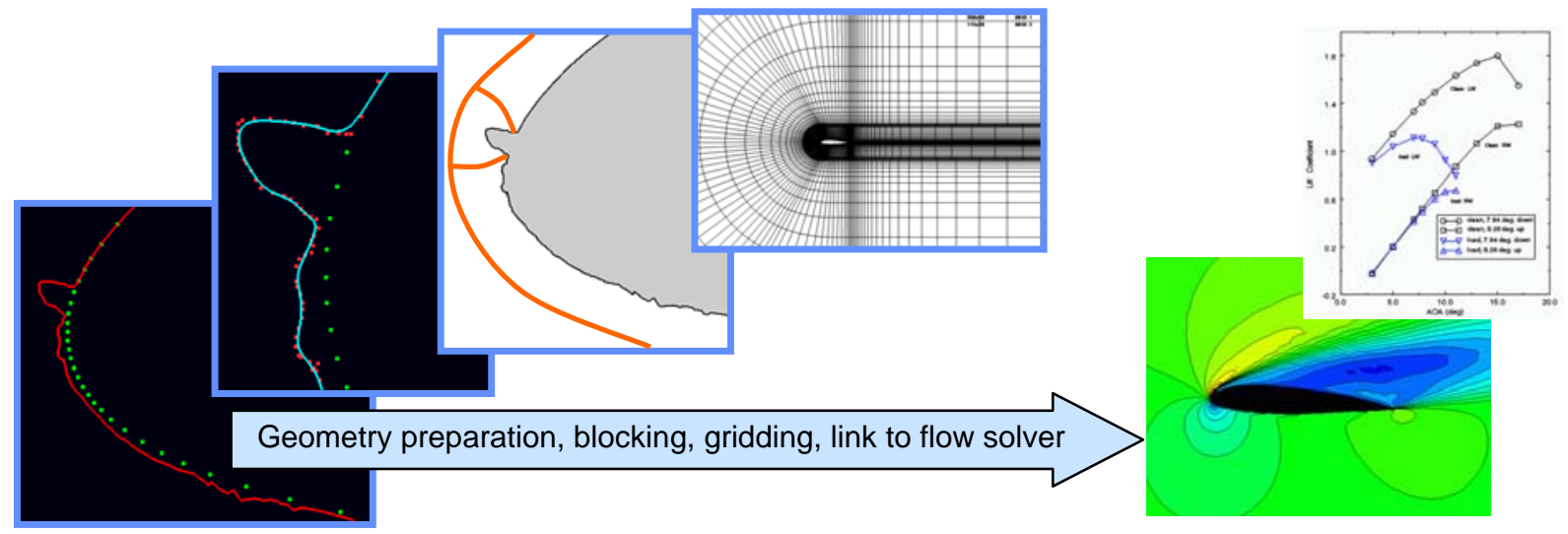

Figure 1: Phases of an Icing Effects Study

controlled to streamline the interactive grid generation process.

SmaggIce was recently expanded with additional capabilities. Version 2.0, the latest release, now accommodates multi-element as well as single-element airfoils for computational studies of icing effects, as shown in Figure 2. Blocking and grid generation for multi-element airfoils required a number of additional supporting capabilities. Version 2.0 automatically creates blocks that connect and fill in the space between elements of a multi-element airfoil. SmaggIce now allows the user to rediscretize boundaries by changing the number and/or distribution of points. These changes are then propagated automatically and recursively to both shared and opposite edges. In addition, the capability to divide blocks arbitrarily between opposite edges and to merge blocks, even if not one-to-one abutting, was

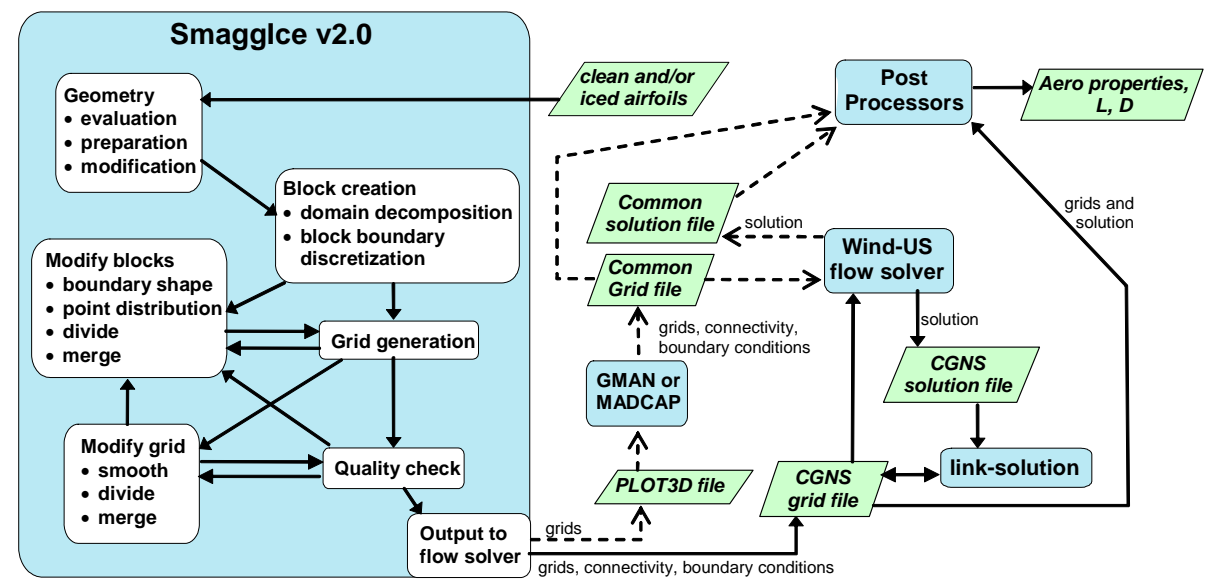

Figure 2. SmaggIce role in icing aerodynamic simulation and analysis process. 
added to the block modification tools. Also, the ability to move the corner points of grid blocks was added.

Grid generation results in a 2D, structured, multi-block grid ${ }^{4,5}$. Grid modification tools in Version 2.0 are now available to stretch and refine blocks and to allow abutting mismatched grids. Evaluating the aerodynamics requires an interface with a flow solver. The interface with the flow solver WIND via the CFD General Notation System (CGNS) protocol was also improved, so that users can proceed easily from grid generation to flow simulation. The graphical interface of SmaggIce is shown in Figure 3, with an example grid created for a multi-element iced airfoil.

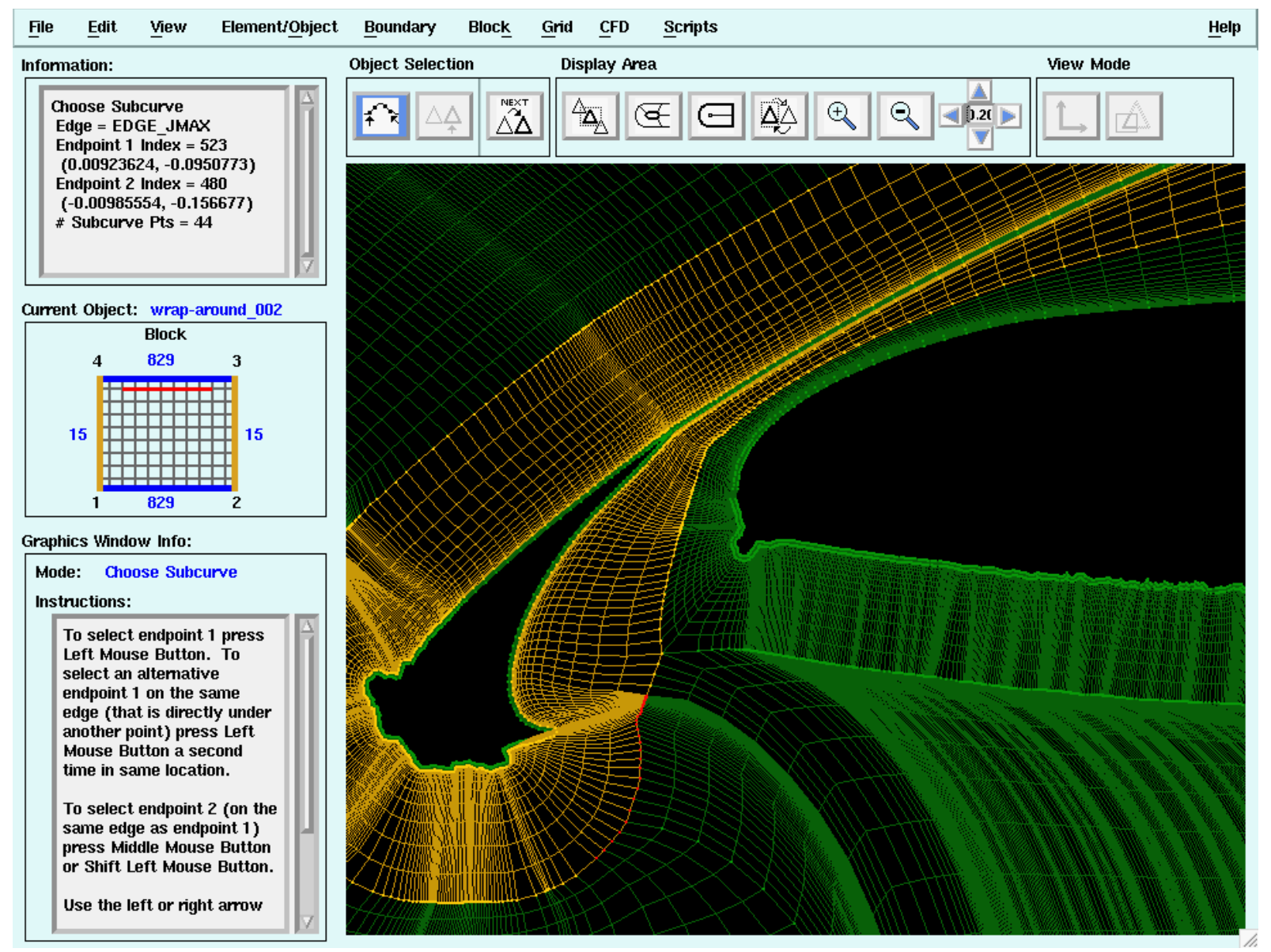

Figure 3. SmaggIce 2.0 Graphical User Interface

\section{Summary of Capabilities}

\section{A. Geometry Preparation}

Tools to prepare an ice shape for gridding are provided in the current version of SmaggIce. Point data defining a clean and/or iced airfoil 2D surface must first be read in, using one of the three common types of input format accepted. Points on the surface of the airfoil should start at the trailing edge but may wrap either clockwise or counter-clockwise. Data may also define an ice shape only, which would include only the points along the ice surface and not the points on the airfoil surface.

Setting a reference airfoil defines the chord length and identifies the leading edge coordinates. These values are needed for several SmaggIce functions, such as normalizing ice measurements or extending ice. A clean airfoil is usually read in and set as the reference airfoil. However, if clean airfoil geometry is not available, an iced airfoil may be used as the reference airfoil, provided the chord length and leading edge point are input.

In order to prepare the 2D surface for gridding, a number of interactive ice shape control features are provided. These tools allow correction of obvious input errors that commonly arise in experimental ice tracings. These control features of SmaggIce not only prepare the ice surface for gridding and CFD flow simulation, but they also allow 
users to correct any deficiencies (e.g., tangles, twists, gaps, too many or too few points) in the input data. The types of functions that can be applied to surfaces are smoothing, shaping, rediscretization and point redistribution. Any subcurve (or the entire curve) of a surface can be selected for interactive ice shape control.

Direct reshaping of the curve is done by dragging control points associated with the curve. Hyperbolic tangent stretching is also supported. Systematic smoothing of the iced surfaces in a controlled manner is accomplished using a control point formulation. With this feature, irregular ice surfaces can be smoothed to a level acceptable for grid generation. Smoothing is controlled by setting the number of control points in constructing curves. Rediscretization provides a means of increasing/decreasing the number of points, distributing the points by curvature, and controlling the uniformity of their distribution.

In some input geometries, the trailing edge of the airfoil may not be closed; i.e., the first and last points on the upper and lower surface at the trailing edge do not coincide. In this case, one of the tools provided can extend the trailing edge of an "open" airfoil element with a single menu selection. For some ice shapes, only the geometry of the ice accretion is included, rather than the coordinates for the complete airfoil. This is also known as an "open" geometry, one in which the coordinates do not include the complete airfoil, but only the point for the ice accretion around the leading edge. Open geometries are frequently obtained from experimental tracings. In this case, the user may read in the clean airfoil on which the ice is based and SmaggIce will automatically extend the ice to include the clean airfoil before creating a grid.

SmaggIce can also examine airfoil and ice geometry when it is read in and after modifications are made to it, to search for and identify twists and/or tangles in the surface. These twists will be visually highlighted on the screen, so that they can be corrected either manually or automatically. This is shown in Figure 4.

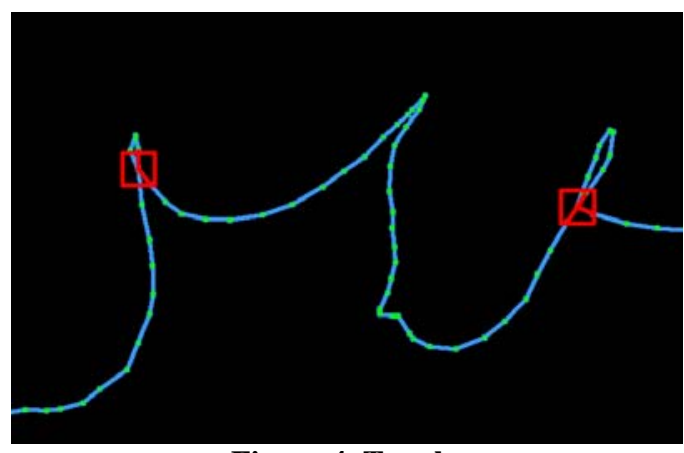

Figure 4. Tangles

\section{B. Domain Decomposition}

The basic blocking strategy and topologies used in SmaggIce will result in high-quality multiple-block grids for 2D iced airfoils. Methods and guidelines for generating high-quality multi-block Navier-Stokes grids, and strategies to provide fast convergence, have been incorporated in the development ${ }^{5}$.

The first step in domain decomposition of a single-element airfoil is to define a wake, which extends downstream from the trailing edge of the iced airfoil. The wake is used to specify the cut for the C-shaped topology that must be used during domain decomposition. The number of points, the length, and the angle of the wake can all be set manually, or using typical default values. The spacing of the wake points closest to the trailing edge is based on the spacing of points on the airfoil at the trailing edge. The rest of the point spacings are set to accommodate the specified length and number of points.

Domain decomposition divides the flow domain into blocks, and is performed prior to gridding. The domain is decomposed in steps, with various types of blocks created during each step. For single-element airfoils, the domain consists of three or more blocks- a viscous sublayer, one or more near field blocks, and

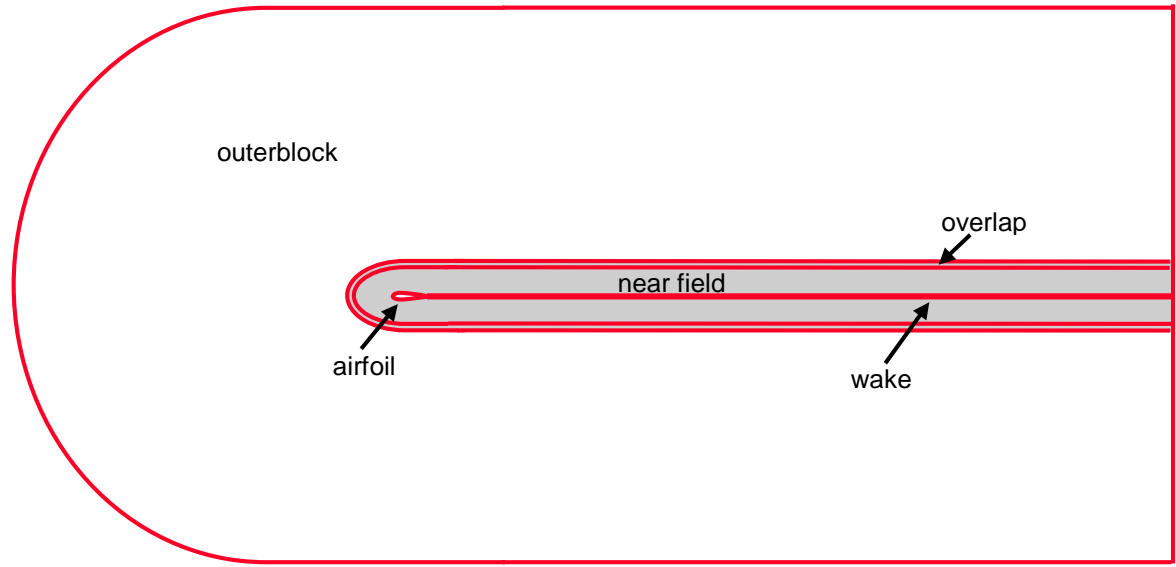

Figure 5. Single-element Blocking Strategy. an outer block that overlaps the near field. This is shown in Figure 5. 
The viscous sublayer block is a thin C-shaped domain that wraps around the wake and iced airfoil. It serves as a transition from the irregular geometry of the iced surface to a smoother outer boundary while simultaneously providing a very dense mesh near the no-slip boundary with firm control. The points along the inner boundary of the viscous sublayer block are automatically created using the points along the wake and iced airfoil. The spacing of the points in the radial direction is specified by three parameters: (1) the number of points in the radial direction, (2) the thickness of the block, and (3) the initial grid spacing adjacent to the iced airfoil and wake. Concave areas of ice can introduce tangles in the viscous sublayer block. These tangles are automatically indicated, and can be removed by reducing the thickness of the block, manually smoothing the ice surface before creating the block, or using an automatic process to smooth out the inner and outer edges of the viscous sublayer block. An example of this feature is shown in Figure 6. When the viscous sublayer domain decomposition is complete, the viscous sublayer block is created and a grid is generated for that block.

The user selects the basic blocking topology to be used when creating the near field. Either single block or multiple blocks may be used. For clean airfoils, or airfoils with relatively simple rime ice shapes, a single block topology may be sufficient. For more complicated ice shapes, multiple blocks are recommended. In the case of airfoils with prominent glaze horns, the local flow field is dominated by separated flows aft of the suction-side horn. In such cases, the point of flow separation at the horn tip is critical to predicting the airfoil performance, but detailed geometry of other parts of the ice may not be as critical. The near field block(s) allow for easy accommodation of complex geometric features. The multiple block topology is based on userdefined radial cuts. SmaggIce allows the user to interactively select and move the endpoints of the radial cuts, then shape the cuts.

The inner boundary of the near field is automatically defined as abutting one-to-one with the outer boundary of the viscous sublayer block. The initial grid cell spacing along that inner boundary is set to match the last cell spacing along the outer boundary of the viscous sublayer. The outer boundary of the near field consists of a semi-circle centered on the leading edge point and a parallelogram abutting the semi-circle and extending to the end of the wake. Parameters are specified to set the radius of the leading edge semi-circle, the number of points in the radial direction, and for radial cut topology, the number of radial cuts to use. When the near field domain decomposition is complete, the near field blocks are created and grids are generated for each of those blocks.

The user also defines parameters for generating the outer block. The outer boundary of the outer block consists of a semi-circle centered on the leading edge point and a rectangle abutting the semi-circle and extending to the end of the wake. The inner boundary is

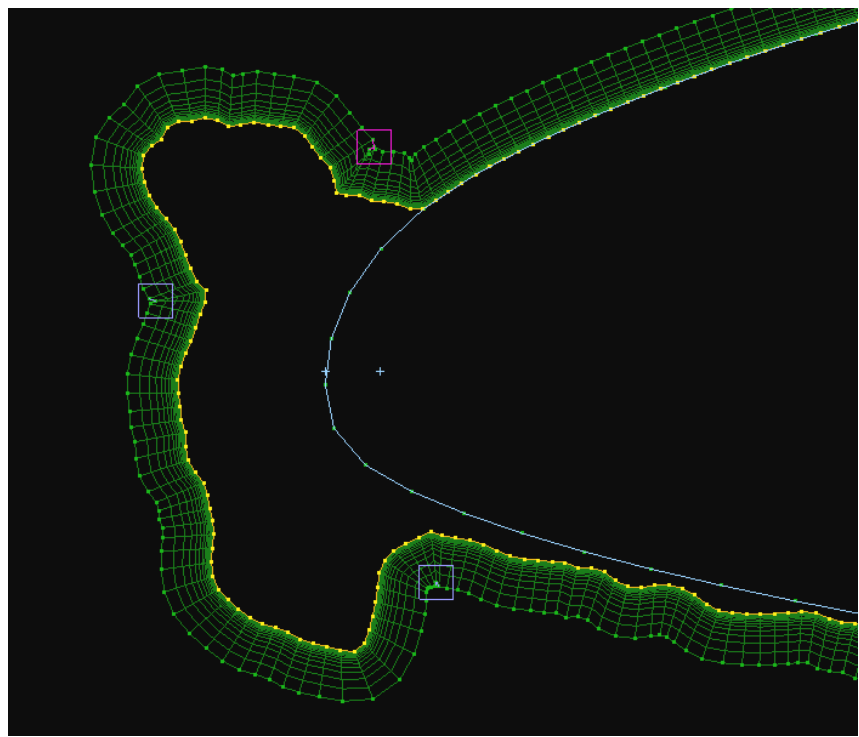

Figure 6. Twists and Creases Highlighted in Viscous Sublayer Boundary

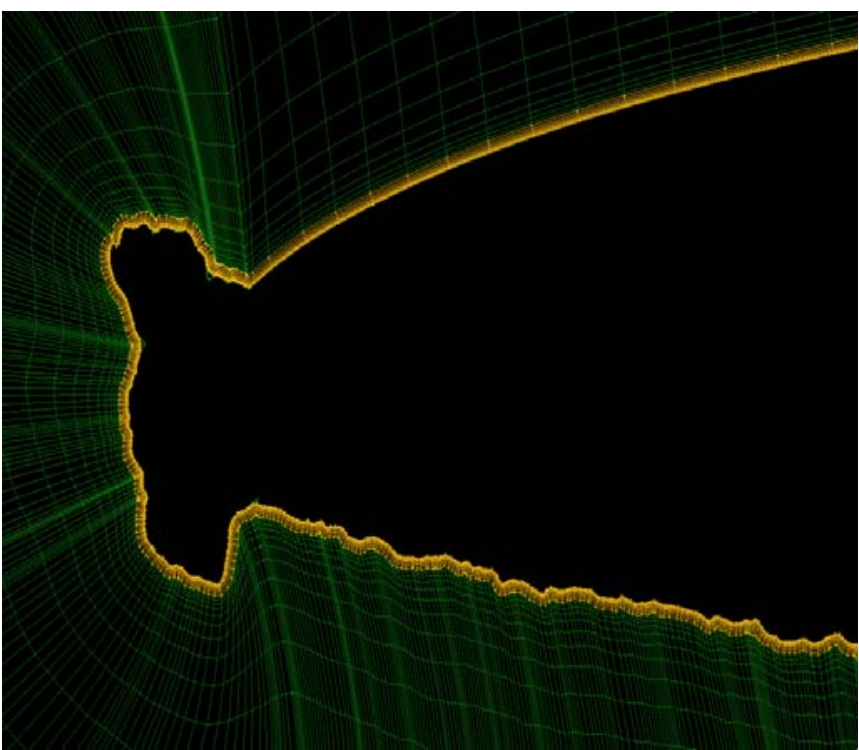

Figure 7. Viscous Sublayer and Near-Field Blocks created to overlap the outer boundary of the near field. The user specifies the number of cells to overlap, the number of points in the radial direction and the azimuthal direction of the grid, and the outer radius of the semi-circle at the leading edge. After these parameters are specified, the boundaries of the outer block and its grid are generated. 
Appropriate boundary conditions are assigned to block boundaries during domain decomposition. SmaggIce can accommodate either no-slip or Euler boundary conditions along the surface of the airfoil, as shown in Figure 8. The viscous sublayer block and near field blocks are created with one-to-one abutting connectivity to each other, while the near field blocks and the outer block have overlapping (overset) connectivity between them. These connectivities are stored for each block, along with the geometry. When modifications are made to block boundaries (rediscretizing, redistributing points, dividing and merging grids), the connectivity information is maintained or modified as necessary. Inverse bilinear interpolation is used to compute the overlapping connectivities at boundary points of the outermost near field blocks that overlap the outer block and at the boundary points of the outer block that overlap near field blocks. An efficient and robust algorithm was developed that uses fast algebraic calculations rather than an iterative procedure. The algorithm identifies and properly handles every possible degenerate special case of a quadrilateral cell.

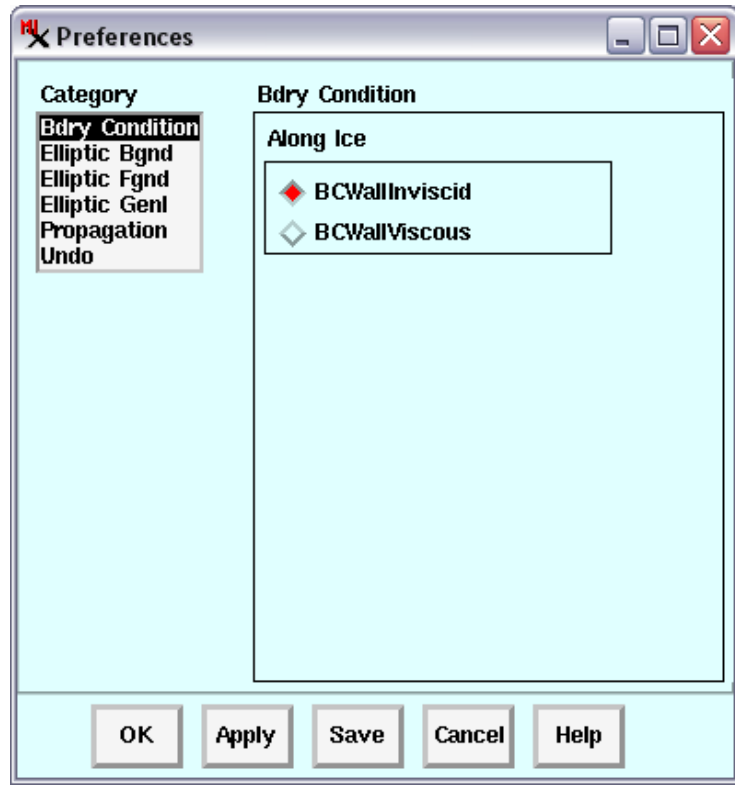

Figure 8. Airfoil Surface Boundary Interface

For a single-element airfoil, the required steps for initial domain decomposition and grid generation are:

- Identify the clean airfoil (or at least specify the chord length and the leading edge point).

- $\quad$ Select airfoil on which the grid will be based.

- $\quad$ Create a wake.

- Create a viscous sublayer block. This is a required, C-shape block, which wraps around the airfoil and extends back along the wake.

- $\quad$ Create the near field block(s). This consists of at least a single block that surrounds the viscous sublayer block, but it may be defined as multiple blocks with radial cuts dividing them.

- $\quad$ Create the outer block which overlaps the near field block(s).

The same tools that are used for geometry preparation also can be used to modify block boundaries, including the radial cuts that make up the near field in multiple-block topologies. This is a powerful feature of SmaggIce which allows rapid, interactive shaping of the overall topology to ensure a quality final grid. After a block has been created, the shape of the block boundary can be adjusted. Control points along the subcurve of the boundary can be dragged using the mouse, as shown in Figure 9. When the boundary is modified, the grid is removed, since it no longer matches the boundary. A preview of the adjusted points

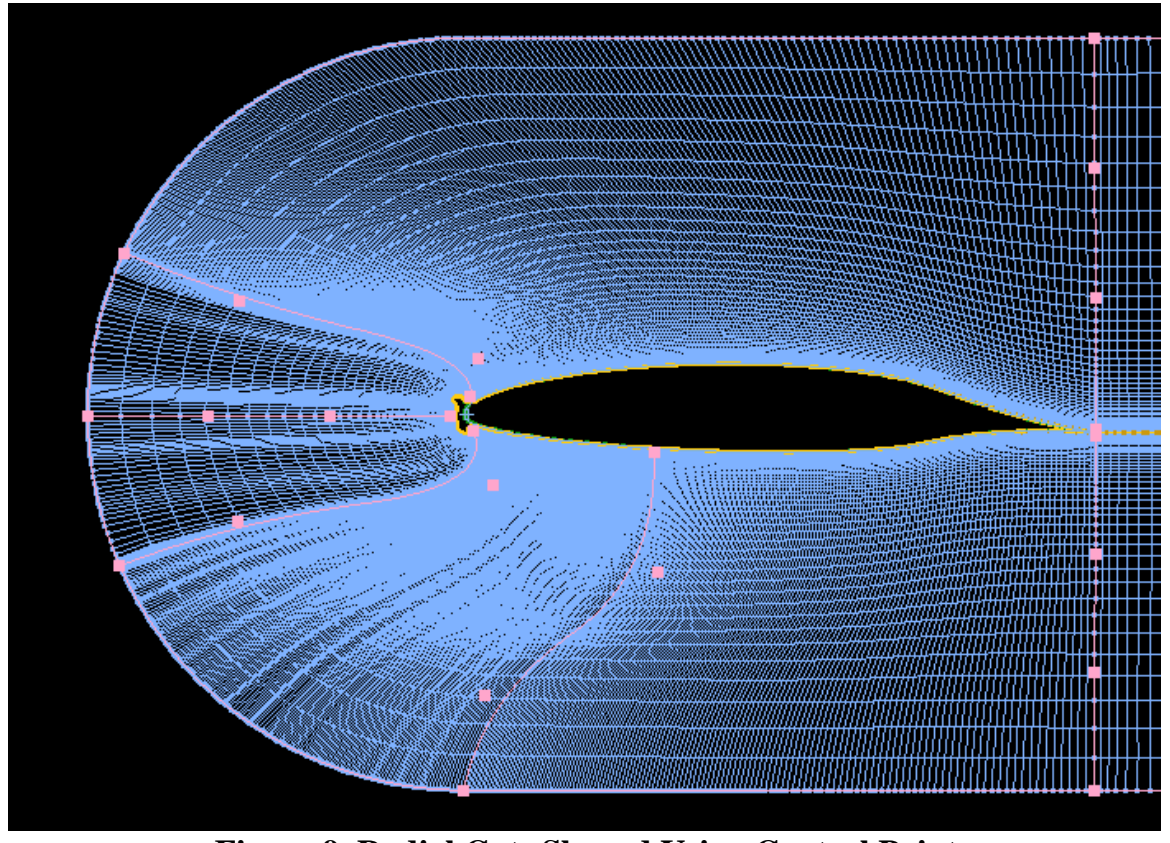

Figure 9. Radial Cuts Shaped Using Control Points is then displayed. When the changes are applied, the abutting boundary from the adjacent block is automatically changed to keep the 
connectivity intact. Then, grids are automatically regenerated for the modified blocks. Frequently, points need to be distributed along a block edge so that the cell spacings match those of the block's neighbors. SmaggIce provides a tool to do this. The spacing can match at either end or both ends of a block edge.

\section{Multi-element blocking}

Four methods were initially considered for multi-element blocking and grid topology. The "wake connectors" was the simplest conceptually, but it was found that it was hard to increase grid density in some areas, and hard to get grids in the small gaps between elements. Another method ${ }^{6}$, allowed for all structured, all abutting one-to-one blocks. This method would have created many blocks which would quickly become tedious to modify with an interactive tool. A hybrid method ${ }^{7}$ considered was very different from the abutting block approach used by SmaggIce, and therefore was not suitable. What was chosen was a method incorporating separate wakes off of each element ${ }^{8}$.

Domain decomposition divides the flow domain into blocks, as with single-element blocking, and is performed prior to gridding. The domain is likewise decomposed in steps, with various types of blocks created during each step. For multi-element airfoils, the domain consists of multiple blocks: one block for each element, one wrap-around block for each element that surrounds the viscous sublayer blocks and connects them together, one or more near field blocks, and a single outer block.

This method required several major changes to SmaggIce in order to implement it. The major issues were as follows: (1) multiple wakes must be saved and associated with blocks, (2) multiple viscous sublayers will exist and be associated with blocks, (3) there will be another type of block for the wrap-around block- in addition to the viscous sublayer, near-field and outer-block types, and (4) the near field will connect to multiple wrap-around blocks, not just a single viscous sublayer.

As with the single-element case, the user must first identify the clean airfoil, or at least specify the chord length and the leading edge point. Then, set
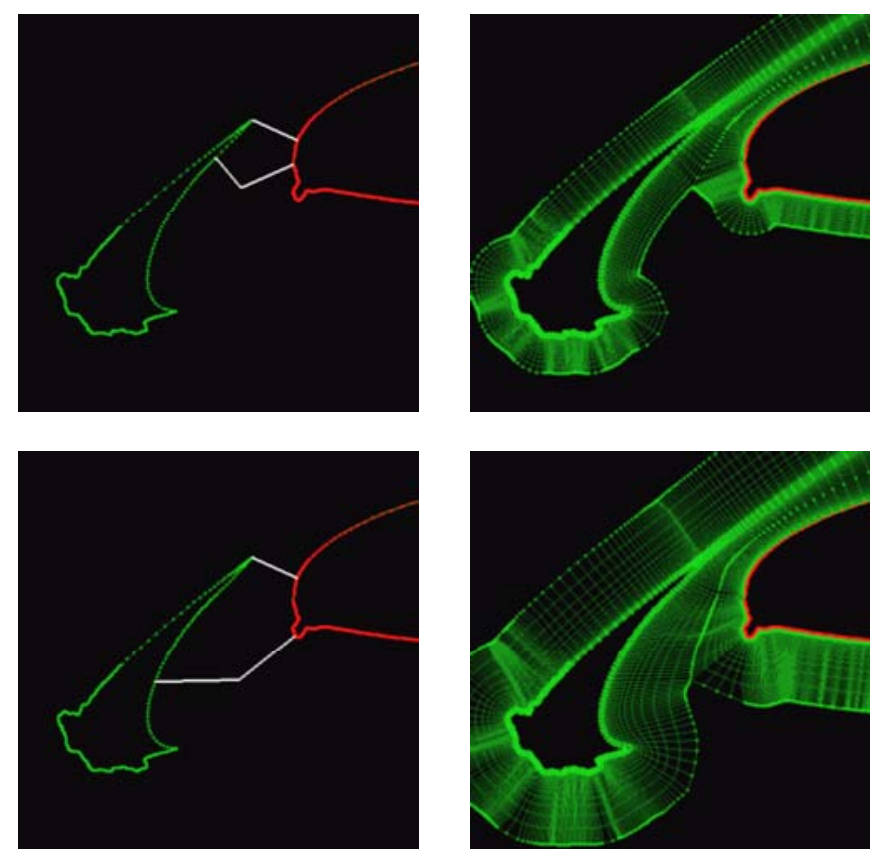

Figure 10. Creating Links Between Elements

the reference airfoil as in previous versions. Following this, links must be created between the elements that make up the multi-element airfoil, as shown in Figure 10. These links are created one at a time, starting with the rightmost element of the airfoil. It is important to create good links between the elements in a multi-element airfoil, because the locations of the lines that define the links will affect the shape of the wrap-around blocks between and around the elements. The positions of the points of the lines that define each link can be moved.

Next, wakes for each element in the multi-element airfoil must be generated. The user enters the parameters for each wake, and is given a preview wake before being asked to confirm the desired shape. The system then automatically propagates the number and distribution of points downstream and along the upper edge. The user then has the option to create critical points for each wake, allowing the wake to be reshaped so as not to interfere with other parts of the geometry. This is shown in Figure 11.

Once the wakes have been defined, the viscous sublayer and wrap-around blocks must be created for each element. The block for each element of the airfoil contains the Navier-Stokes viscous sublayer for that element. As in the single-element case, the viscous sublayer blocks are C-shaped blocks that surround each element and extend back along the wake.

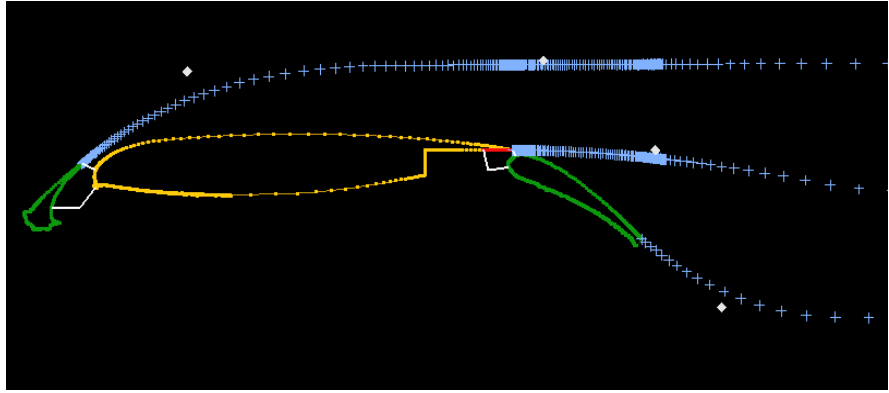

Figure 11. Wake Elements Shaped Using Critical Points

Much of this process is automated, in part to 
ensure that the thickness of the viscous sublayer is not greater than $25 \%$ of the smallest distance between elements. The wrap-around blocks are C-shaped blocks that wrap around each viscous sublayer block and fill in the space between them, as shown in Figure 12.

Next, the near field block(s) are created. This step requires at least one block that surrounds the wrap-around blocks, but it may be defined as multiple blocks with radial cuts dividing them. Finally, the outer block which

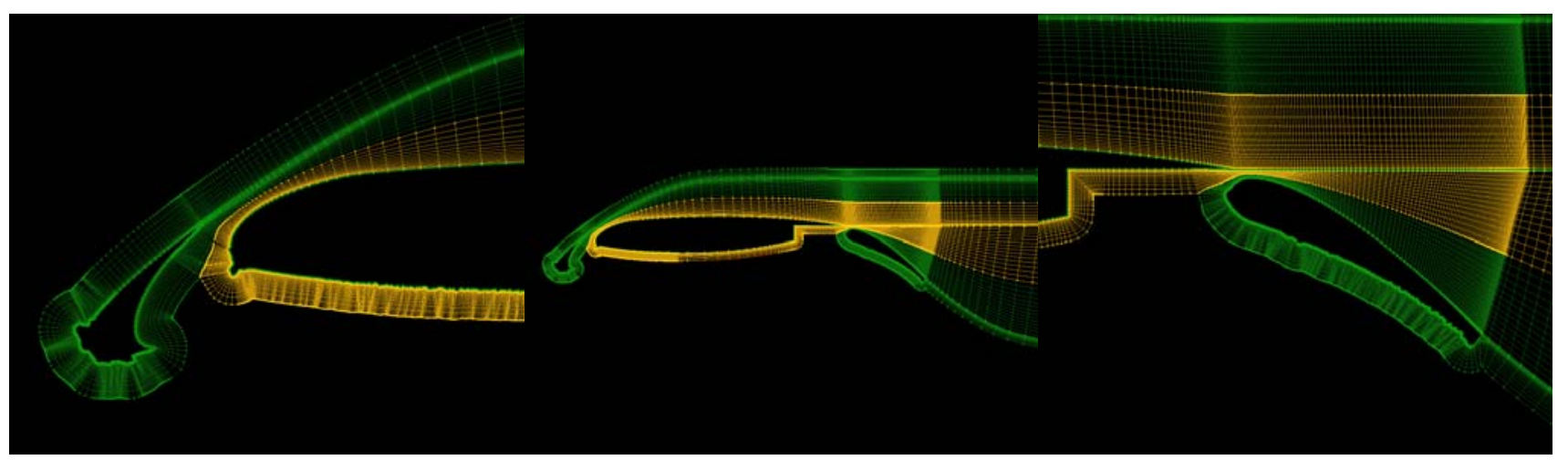

Figure12. Sublayer and Wrap-around Blocks for a Multi-Element Airfoil

overlaps the near field block(s) must be created. This may be easily shaped, if desired, using radial cuts as shown in Figure 13. The complete blocking of a multi-element airfoil is shown in Figure 14.

For a multi-element airfoil, the required steps for initial domain decomposition and grid generation are:

- Identify the clean airfoil (or at least specify the chord length and the leading edge point).

- Create links between the elements that make up the multi-element airfoil.

- Create the wakes for each element in the multielement airfoil.

- Create the viscous sublayer and wrap-around blocks for each element.

- Create the near field block(s).

- Create the outer block which overlaps the near field block(s).

SmaggIce also includes a feature for moving airfoil elements, with which to modify elements by rotating and/or translating the geometry. For instance, an aileron element can be translated and rotated about a user-specified hinge point. This may be useful to prepare for parametric studies on multi-element configurations. This feature is shown in Figure 15.

\section{Grid Generation}

To generate its grids, SmaggIce uses transfinite interpolation followed by grid smoothing. The transfinite interpolation is done using a routine from a gridding package provided by NASA Ames Research Center.

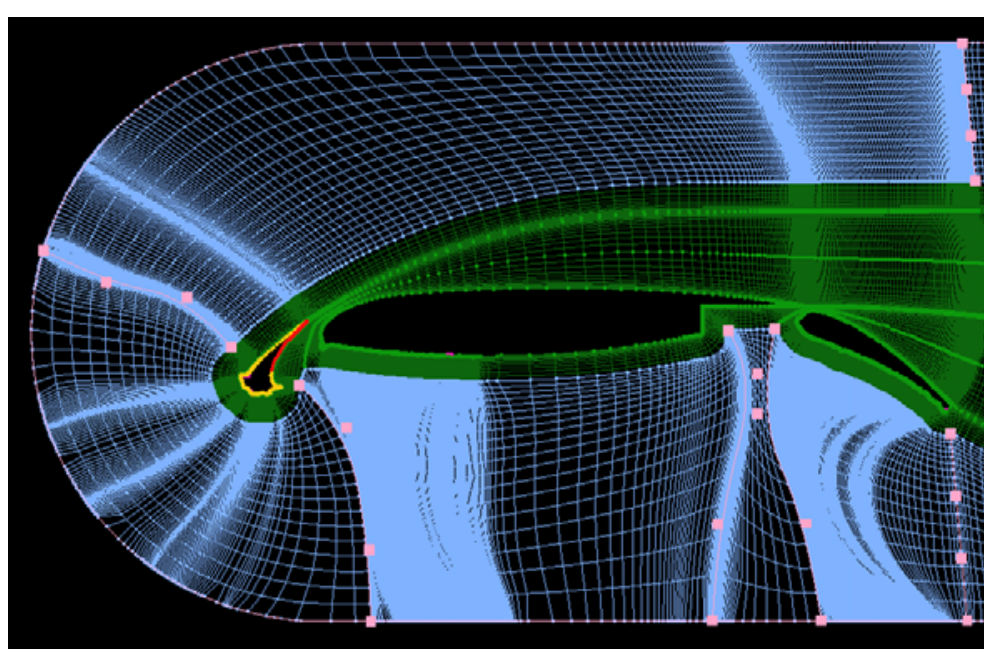

Figure 13. Near-Field Blocks Shaped Using Radial Cuts

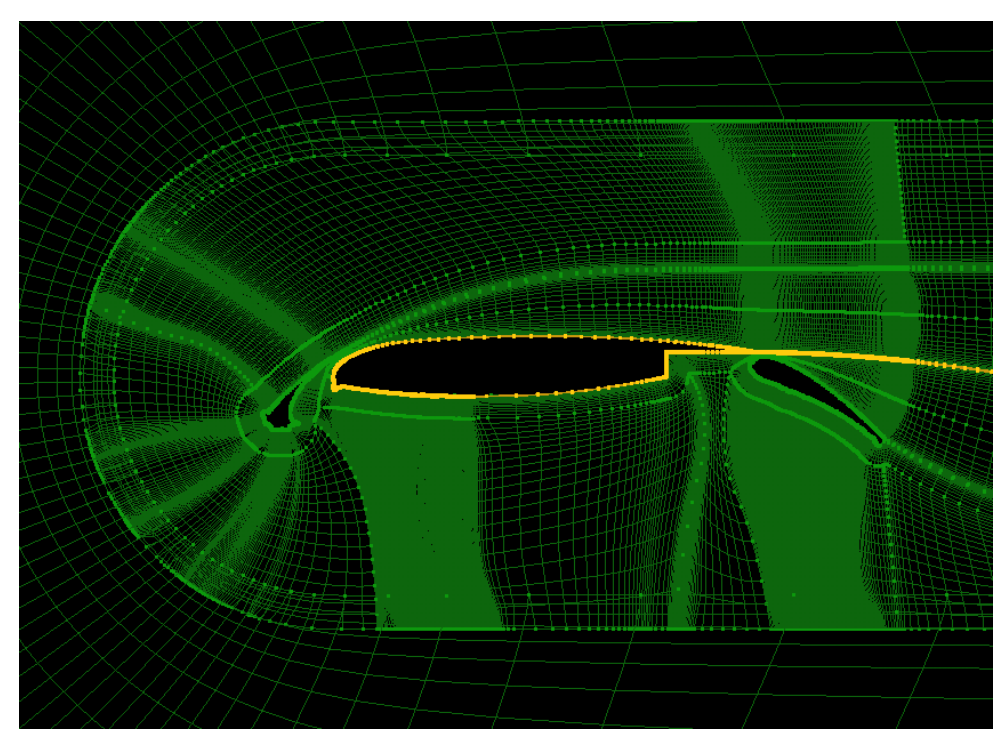

Figure 14. Complete Blocking of Multi-Element Airfoil 
SmaggIce can perform two kinds of grid smoothing. Elliptic smoothing is done using a variant of Thomas Middlecoff background control functions. Several parameters to the elliptic smoothing function, such as number of iterations and tolerance, can be modified by the user. The user also has control of elliptic smoothing parameters used during grid generation. Changing these parameters will affect the appearance of the grid.

The second kind of smoothing smoothes across two abutting blocks. The user first selects a subcurve on the shared boundary between the blocks. A sub-grid consisting of grid cells from both blocks on either side of the subcurve is then smoothed using Laplace smoothing. This smoothing necessarily modifies the location of the selected subcurve.

Other tools which allow the user to modify grids include dividing a gridded block, merging two gridded blocks which are abutting one-to-one, and copying the spacing of points along one edge of a gridded block to the interior grid lines.

After the initial domain decomposition and grid generation, block boundaries may be modified to change the shape of the boundaries or change the point distribution. The number of points may not be changed at this time. This is to ensure that all blocks which are grid-ready and one-to-one

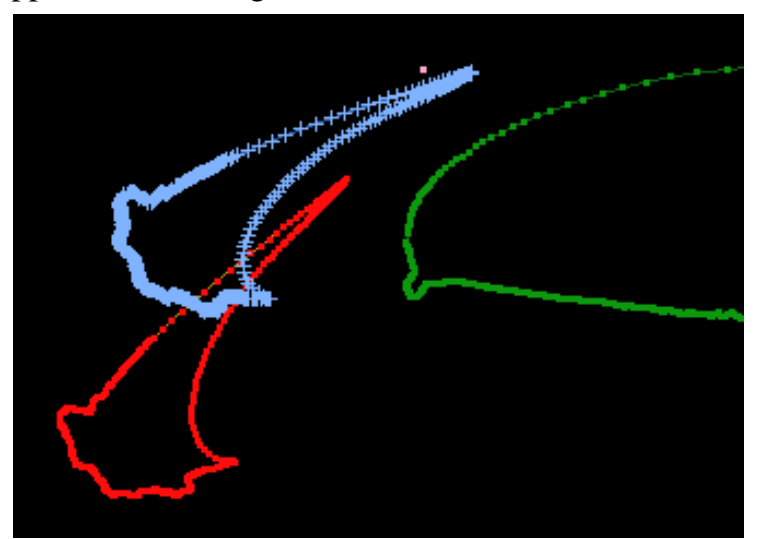

Figure 15. Geometry Translation and Rotation abutting remain so even after modifications are made.

Grids may be modified by:

- $\quad$ dividing along grid lines

- $\quad$ dividing along arbitrary lines across opposite edges of the block; these lines may be re-shaped

- $\quad$ merging one-to-one abutting grids or blocks that share corners

- $\quad$ smoothing grids across shared edges of blocks

- $\quad$ performing interior smoothing

- $\quad$ setting cell spacing along an edge to match cell spacing of adjacent grids.

Blocking and grid generation for multi-element airfoils required a number of additional supporting capabilities. Version 2.0 allows the user to rediscretize boundaries by changing the number and/or distribution of points. These changes are then propagated automatically and recursively to both shared edges and opposite edges, as shown in Figure 16 via the user interface in Figure 17. In addition, the capability to divide blocks arbitrarily between opposite edges was added to the block modification toolkit.

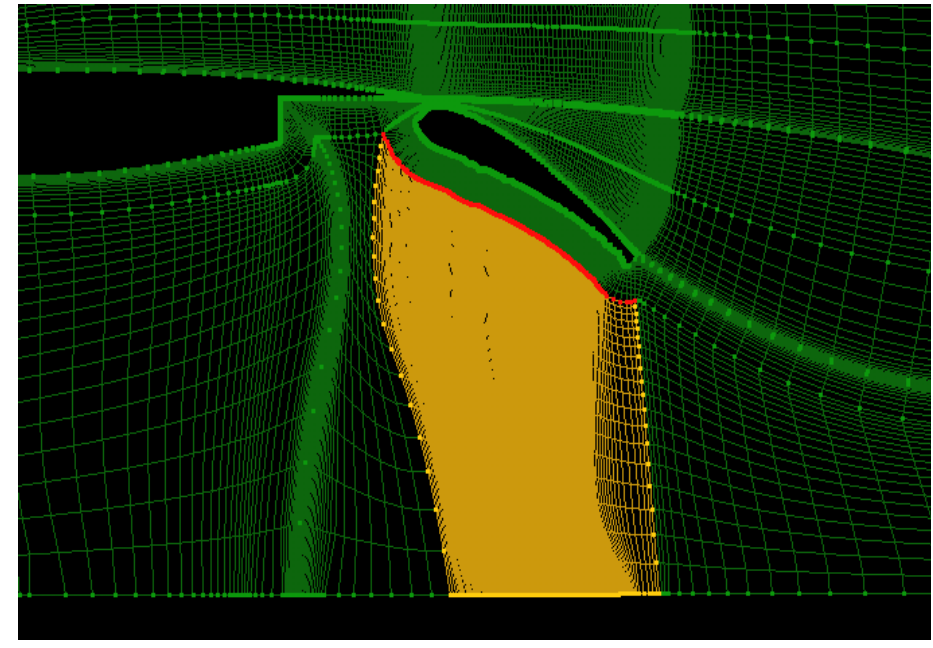

Figure 16. Re-gridding of Near-field Block

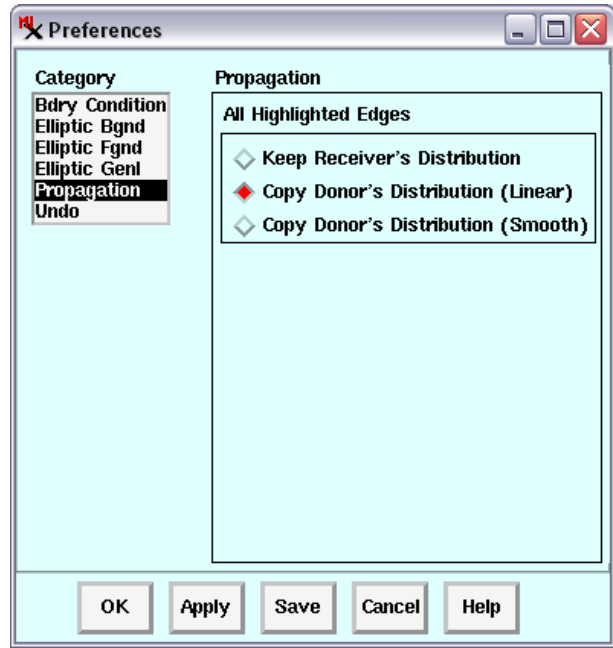

Figure 17. Propagation Interface 


\section{E. Grid Quality Analysis}

Grid quality measurements can be calculated and displayed graphically on the gridded blocks as color-coded overlays. This allows the user to quickly identify areas of poor grid quality, which may then be repaired. Minimum and maximum values for these measurements are also displayed. Examples are shown in Figures 18 and 19.

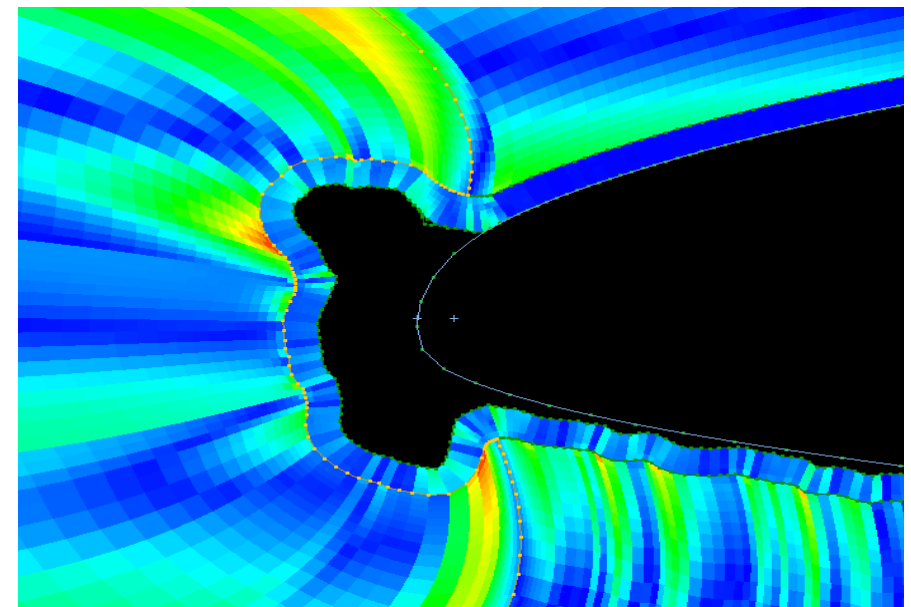

Figure 18. Grid Quality (Orthogonality)

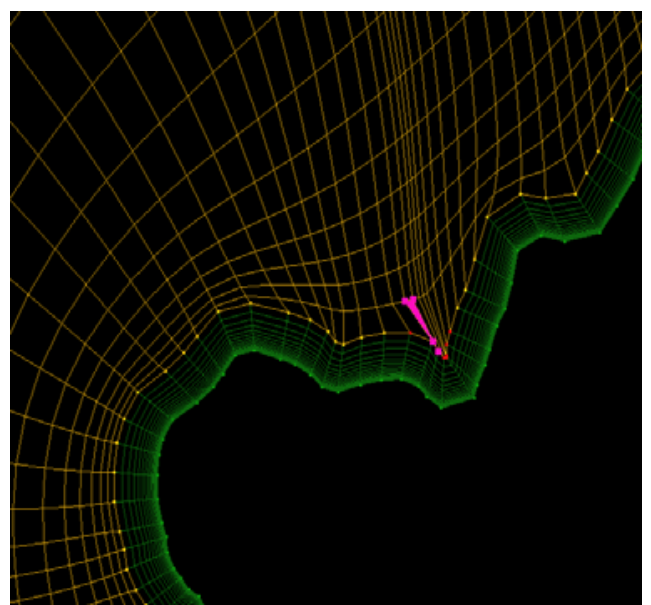

Figure 19. Invalid Cell Shapes

These grid quality measurements include:

- $\quad$ aspect ratio of grid cells

- $\quad$ orthogonality of grid cells

- $\quad$ skewness of grid cells

- $\quad$ stretching ratio in the I direction at each grid point

- $\quad$ stretching ratio in the $\mathrm{J}$ direction at each grid point

- $\quad$ grid cell shape (twisted, concave, or degenerate cells)

- $\quad$ tangled grid using value of cell Jacobian

\section{F. Output}

When saving to a CGNS file, all boundary conditions are stored along with the geometry for each block and output with the geometry. The connectivity information is also output with the geometry when saving to a CGNS file. This saves the user the extra steps of having to use a separate program to define the boundary conditions and the connectivities between blocks. An alternate format for output, compatible with PLOT3D, while more generic, does not retain either the boundary or connectivity information. This format allows for rapid import of SmaggIce grids into a wide variety of grid generation and post-processing software, however. Certain geometries may also be saved in a point cloud format, which retains only the $\mathrm{x}, \mathrm{y}$ and $\mathrm{z}$ coordinates of surface points.

Output files generated by SmaggIce in the CGNS format are compatible with the WIND flow solver. This is another new feature that has been added for version 2.0. The use of CGNS as a file format to transfer information to and from other CFD applications like post-processors and flow solvers has been a long-term goal of the SmaggIce project. Using the CGNS format allows ice geometry, grids, boundary conditions, connectivity and solutions to all be saved in a single file. Additionally, SmaggIce offers a simple tool to create a generic input file for the WIND flow solver, in addition to the grid file.

\section{G. Ice Shape Characterization}

Parameters like upper horn thickness and ice mass are widely used by researchers and analysts in performing sensitivity studies. Sensitivity tests ${ }^{9}$ are used to characterize the influence of small changes in environmental or tunnel parameters on aerodynamic effects. They provide a link between spray cloud, ice changes and aerodynamic effects, and allow designers to develop preliminary estimates that a minimal change in LWC or MVD would have on aerodynamic characteristics.

The ice shape characterization tools incorporated in SmaggIce are used to measure and record location, length, angle, arc length, and ice area. This gives users the means to measure the physical characteristics of ice such as icing limit locations, horn height and angle, distance along the clean airfoil from the leading edge to a prominent ice 
location, or the area of ice between two user-specified points. These tools provide an aid in the process of determining the relationship between ice characteristics and their effects on aerodynamic performance.

Ice shapes can be measured interactively by selecting data points on the screen, recording the measurements in a table, and saving them to a file. In SmaggIce, an airfoil is defined as a sequence of points, i.e., (x,y) coordinates, starting at the upper (or lower) surface of the trailing edge and following along the surface toward the leading edge and then back toward the trailing edge along the lower (or upper) surface. Ice shapes are also defined by a sequence of points, although they do not have to include points back to the trailing edge. All modifications to the geometry are made to these points.

The types of measurements that can be made include:

- $\quad$ point location (e.g., ice limits)

- $\quad$ distance between two points (e.g., ice horn height or width)

- $\quad$ arc length

- $\quad$ angle between two lines (e.g., horn angle)

- $\quad\left(\mathrm{X}-\mathrm{X}_{\mathrm{le}}\right) / \mathrm{C}(\mathrm{e} . \mathrm{g} .$, distance from leading edge $)$

- $\quad$ ice area

Location and distance may be normalized by the chord length of a clean airfoil. Ice area may be normalized by the area of the clean airfoil. The points used in making the measurements can be selected by various methods: the closest point to any object, the closest point to the currently-selected object, the closest point to the reference airfoil, or an arbitrary point in space.

\section{H. Create and Place Artificial Ice}

Adding computer-generated ice shapes to the surface of a clean airfoil is used to prepare for studies of the effects of surface roughness as well as the effect of various ice shapes on the airfoil aerodynamic performance ${ }^{10}$.

Once clean airfoil data is read in, the user may interactively add different types of geometries to the clean airfoil. A tool is provided to attach artificial (i.e., computer-generated) ice shapes such as forward-facing right triangle, backward-facing right triangle, generic triangle, rectangles, forward-facing quarter circle, backward-facing quarter circle, half-circles, and trapezoids to the surface of an airfoil. Parameters for location, replication, size, and number of points can be specified. This tool facilitates studies of the effects that various ice shapes and ice roughness have on aerodynamic performance. An example of artificial ice is shown in Figure 20.

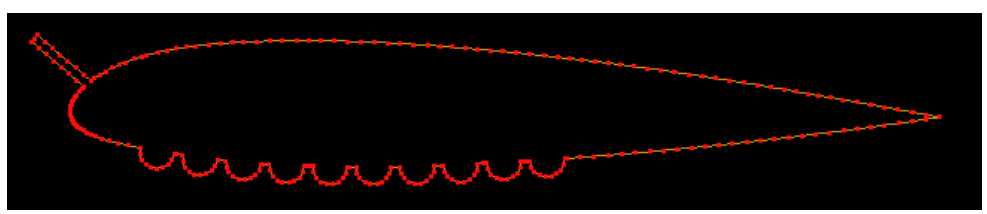

Figure 20. Artificial Ice Added to Clean Airfoil Geometry

\section{Extruded 3D Wings}

While SmaggIce is not intended as a fully three-dimensional tool, some limited 3D capabilities have been implemented. Airfoil geometry can be extruded to generate a surface grid of a three-dimensional wing. Sweep angle is an available parameter and no tip shapes are generated. When both a clean and iced airfoil section are defined, SmaggIce can generate a three-dimensional wing with ice that varies along the span through the application of sinusoidal variations in the spanwise direction. The number of cycles across the span and the amount of variation are available input parameters. The interpolation between the clean shape and the iced shape as a function of span is automatically performed.

\section{J. Convenience Features}

SmaggIce incorporates an array of features to improve its convenience and usability. Most notable among these is the undo/redo feature. SmaggIce has full undo and redo capability for all user actions that affect the data defining elements, wakes and blocks. The number of undo/redo levels can be set by the user. The toolkit also includes a save state feature, which saves all current information about elements, blocks, grids and settings to a file. A restore state command allows previous sessions to be loaded.

A scripting interface is provided to allow frequently-performed commands or sequences to be repeated. Scripting enables the recording feature of SmaggIce, which records all of the user's inputs to an easy-to-read text file. Recorded scripts are text files and can be easily edited. 
Extensive help files can be displayed from within the SmaggIce interface, or can be viewed using a Web browser. A detailed user manual along with a small number of tutorial cases is distributed along with the code.

\section{Software Development}

Some challenges facing the software development team included the requirement for multiple platforms, integrating code from different contributors, different languages (mostly C, some FORTRAN), and a limited budget. For these reasons, the development of SmaggIce relied heavily on the use of open source tools.

The libraries used included:

- $\quad$ iniParser, a C library for maintaining preference files

- $\quad$ Python, an embedded interpreter enabling scripting of SmaggIce via Python

- $\quad$ CGNS, a library for storage and retrieval of CFD data

- Libmba, a general purpose C library including a CSV file parser

The open source tools used included:

- Bugzilla, a bug tracking system

- $\quad$ CVS, a revision control system

- $\quad$ DDD, a graphical front-end for command-line debuggers such as GDB

- $\quad$ Splint, for statically checking $C$ program coding

- $\quad$ gcc, a cross-platform C library

- $\quad$ GNU Make, a cross-platform build tool

- $\quad$ SWIG, which wraps $\mathrm{C}$ code so that it is callable by Python

- Doxygen, a source code documentation generator tool

- Cscope, a developer's tool for browsing source code

The SmaggIce code does some inline "self-checking". A routine called SmgSanityCheck is called after each user action. It checks to make sure that the state of SmaggIce is consistent and within expected bounds. If an error is detected, SmaggIce aborts. SmaggIce also makes use of some common software design patterns. In particular, the Command Pattern was used to implement undo/redo and the scriptability and recordability of SmaggIce. The recordability feature is used to log all user actions. This allows the user to re-run their session via a script. This is useful if, for example, SmaggIce aborts for any reason.

The development approach was chosen so it could be steered by the customer. This allowed changes and additions to be made quickly when new requirements were discovered as development progressed. Continuous integration with an always-available working code and group ownership of source code helped keep development on schedule. SmaggIce was designed and written to run on any UNIX platform. It has been ported to run on MSWindows systems using third party X-server software available from various sources.

\section{Future Plans}

In the future, SmaggIce will be integrated into the NASA GlennICE computational environment, which is currently still being defined. Additional capabilities being considered include a quasi-3D approach for aerodynamic performance, enhanced block and grid modification tools, grid error estimation, and further integration between flow solvers and multi-disciplinary tools. Ultimately, icing effects studies will have to go to fully 3D and possibly unstructured grids. Plans are to continue supporting primarily Windows PC and Linux platforms. SmaggIce can also be used for grid generation of airfoils without ice accretion.

\section{Conclusion}

SmaggIce was recently expanded with additional capabilities. SmaggIce is one of NASA's aircraft icing research codes, used for aerodynamic performance prediction of iced airfoils. Its primary purpose is to create high-quality structured grids for two-dimensional iced airfoils in preparation for computational fluid dynamics analysis. It provides tools to measure ice shape characteristics, prepare the ice surface for gridding, perform domain decomposition, create and modify grids, analyze grid quality and output grids for subsequent input into aerodynamic flow solvers. Version 2.0, the latest release, now accommodates multi-element as well as single-element airfoils for computational studies of icing effects. Blocking and grid generation for multi-element airfoils required a number of additional supporting capabilities. SmaggIce provides the tools needed to create high-quality grids for aerodynamic simulation of iced airfoils. 


\section{References}

${ }^{1}$ Vickerman, Mary B., Choo, Yung K., Schilling, Herbert W., Baez, M., Braun, Don C., Cotton, Barbara J., "Toward an Efficient Icing CFD Process Using an Interactive Software Toolkit - SmaggIce 2D,” AIAA Paper 2002380, Jan. 2002.

${ }^{2}$ Vickerman, Mary B., Choo, Yung K., Schilling, Herbert W., Braun, Donald C., Baez, Marivell, Cotton, Barbara J., "SmaggIce: Further Progress in Software for Gridding 2D Iced Airfoils," AIAA Paper 2005-1369, Jan. 2005.

${ }^{3}$ Kreeger, Richard E., and Wright, W., "The Influence of Viscous Effects on Ice Accretion Prediction and Airfoil Performance Predictions,” AIAA Paper 2005-1373, Jan. 2005.

${ }^{4}$ Farrell, Charles A., and Choo, Yung K., "Simulation of Two Dimensional Icing Effects on Airfoil Performance Using Smaggice and WIND,” AIAA Paper 2005-1370, Jan. 2005

${ }^{5}$ Chi., X., Slater, J.W., Shih, T. I.-P., Zhu, B., Addy, H.E., Choo, Y.K., "Computing Aerodynamic Performance of a 2D Iced Airfoil - Blocking Topology and Grid Generation,” AIAA Paper 2002-381, Jan. 2002.

${ }^{6}$ J. Thompson, Z. Warsi and C. Mastin, Numerical Grid Generation: Foundations and Applications, Elsevier Science Publishing Co., Inc., 1985.

${ }^{7}$ Noack, R. W., Steinbrenner, J.P., Collection of Technical Papers, AIAA $12^{\text {th }}$ Computational Fluid Dynamics Conference, Jun. 1995.

8 Rumsey, C. L., Gatski, T.B., "Recent Turbulence Model Advances Applied to Multielement Airfoil Computations,” AIAA Paper 2000-4323, Aug. 2000.

${ }^{9}$ Miller, D., Potapczuk, M., "Preliminary Investigation of Ice Shape Sensitivity to Parameter Variation," AIAA Paper 2005-73, Jan. 2005.

${ }^{10}$ Pan, J., Loth, E., Bragg, M., “RANS Simulations of Airfoils with Ice Shapes,” AIAA Paper 2003-729, Jan. 2003. 


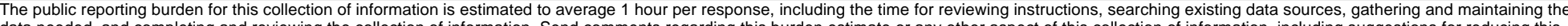

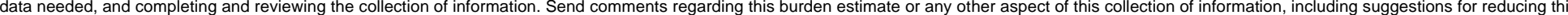

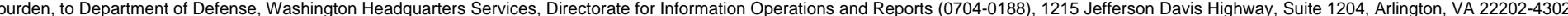

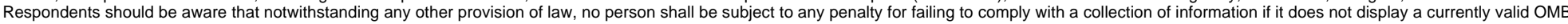
control number.

PLEASE DO NOT RETURN YOUR FORM TO THE ABOVE ADDRESS.

\section{REPORT DATE (DD-MM-YYYY) \\ 2. REPORT TYPE \\ 3. DATES COVERED (From - To)}

01-01-2008

\section{TITLE AND SUBTITLE}

Technical Memorandum

SmaggIce 2.0: Additional Capabilities for Interactive Grid Generation of Iced Airoils

5a. CONTRACT NUMBER

5b. GRANT NUMBER

5c. PROGRAM ELEMENT NUMBER

\section{AUTHOR(S)}

Kreeger, Richard, E.; Baez, Marivell; Braun, Donald, C.; Schilling, Herbert, W.; Vickerman, Mary, B.

\section{5d. PROJECT NUMBER}

5e. TASK NUMBER

5f. WORK UNIT NUMBER

WBS 645846.02.07.03.04.01

\section{PERFORMING ORGANIZATION}

REPORT NUMBER

E-16022

National Aeronautics and Space Administration

John H. Glenn Research Center at Lewis Field

Cleveland, Ohio 44135-3191

9. SPONSORING/MONITORING AGENCY NAME(S) AND ADDRESS(ES)

National Aeronautics and Space Administration

Washington, DC 20546-0001

\section{DISTRIBUTIONIAVAILABILITY STATEMENT}

Unclassified-Unlimited

Subject Category: 02

Available electronically at http://gltrs.grc.nasa.gov

This publication is available from the NASA Center for AeroSpace Information, 301-621-0390

\section{SPONSORING/MONITORS ACRONYM(S) \\ NASA}

11. SPONSORING/MONITORING
REPORT NUMBER
NASA/TM-2008-214829; AIAA-2007-
0502

11. SPONSORING/MONITORING 0502

\section{SUPPLEMENTARY NOTES}

\section{ABSTRACT}

The Surface Modeling and Grid Generation for Iced Airfoils (SmaggIce) software toolkit has been extended to allow interactive grid generation of multi-element iced airfoils. The essential phases of an icing effects study include geometry preparation, block creation, and grid generation. SmaggIce Version 2.0 now includes these main capabilities for both single and multi-element airfoils, plus an improved flow solver interface and a variety of additional tools to enhance the efficiency and accuracy of icing effects studies. An overview of these features is given, especially the new multi-element blocking strategy using the multiple wakes method. Examples are given which illustrate the capabilities of SmaggIce for conducting an icing effects study for both single and multi-element airfoils.

15. SUBJECT TERMS

Aerodynamics

\begin{tabular}{|c|c|c|c|}
\hline \multicolumn{3}{|c|}{ 16. SECURITY CLASSIFICATION OF: } & \multirow{2}{*}{$\begin{array}{l}\text { 17. LIMITATION OF } \\
\text { ABSTRACT } \\
\text { UU }\end{array}$} \\
\hline $\begin{array}{l}\text { a. REPORT } \\
U\end{array}$ & $\begin{array}{l}\text { b. ABSTRACT } \\
U\end{array}$ & $\begin{array}{l}\text { c. THIS } \\
\text { PAGE } \\
U\end{array}$ & \\
\hline
\end{tabular}

18. NUMBER
OF
PAGES
19

19a. NAME OF RESPONSIBLE PERSON STI Help Desk (email:help@sti.nasa.gov) 19b. TELEPHONE NUMBER (include area code) 301-621-0390 

